\title{
Critical Literature 2007: Clinical Topics
}

\author{
Mel L. Anderson ${ }^{1}$ \\ Jeffrey J. Glasheen ${ }^{2}$ \\ ${ }^{1}$ Medical Service, Denver VA Medical Center, \\ Denver, Colorado \\ ${ }^{2}$ Hospital Medicine Section, University of Colora- \\ do-Denver, Denver, Colorado
}

$\mathbf{T}$ his update reviews key clinical articles for hospitalists published over the past year. Selection criteria include high methodological quality, pertinence to hospital medicine, and likelihood that a change in practice is warranted. Table 1 summarizes practice changes.

\section{Enoxaparin Dosing in Acute Coronary Syndromes}

Allen La Pointe NM, Chen AY, Alexander KP, et al. Enoxaparin dosing and associated risk of in-hospital bleeding and death in patients with non-STsegment elevation acute coronary syndromes. Arch Intern Med. 2007;167:1539-1544.

Question: Among patients with non-ST-elevation acute coronary syndromes, how common and harmful is excess enoxaparin dosing? Sponsors: Schering-Plough Corp., Bristol-Myers Squibb/SanofiAventis Pharmaceuticals Partnership, Millennium Pharmaceuticals, and the National Institutes of Health and National Institute on Aging.

Study Design: Observational study of prospective cohort data from the Can Rapid Risk Stratification of Unstable Angina Patients Suppress Adverse Outcomes with Early Implementation of the ACC/AHA Guidelines (CRUSADE) National Quality Improvement Initiative.

Patients: A total of 10,687 patients receiving enoxaparin for nonST-elevation acute coronary syndromes.

Setting: Three hundred thirty-two US hospitals.

Outcomes: Rate of excess enoxaparin dose, defined as greater than $10 \mathrm{mg} /$ day above the recommended dose of $1 \mathrm{mg} / \mathrm{kg}$ every 12 hours for creatinine clearance $(\mathrm{CrCl}) \geq 30 \mathrm{~mL} /$ minute or $1 \mathrm{mg} / \mathrm{kg}$ every 24 hours for $\mathrm{CrCl}<30 \mathrm{~mL} /$ minute; rates of inhospital major bleeding and death; and rate of lower than recommended enoxaparin dose.

Results: Excess enoxaparin dosing occurred in $18.7 \%$ of the cohort $(2002 / 10,687)$. Of these, $57.8 \%(1157 / 2002)$ had $\mathrm{CrCl}<30$ $\mathrm{mL} /$ minute. Excess-dose patients were more likely to be older and female and have a low body mass index $(P<0.001$ for all comparisons). In-hospital major bleeding ( $14.2 \%$ versus $7.3 \%$, $P<0.001)$ and in-hospital death $(5.6 \%$ versus $2.4 \%, P<0.001)$ were more common among excess-dose patients. Enoxaparin underdosing occurred in 29.2\% (3116/10 687) and was not associated with excess harm. Controlling for baseline characteristics, the authors found that the adjusted odds ratio for in-hospital major bleeding in the excess-dose cohort was 1.43 (1.18-1.75, $P<0.001)$ and the adjusted odds ratio for death was 1.35 (1.03$1.77, P=0.03$ ).

Conclusions: Excess enoxaparin dosing in non-ST-elevation acute coronary syndromes occurred in about 1 of every 5 patients treated 
TABLE 1

Summary of Practice Changes from the Reviewed Literature

Start
Dosing enoxaparin on the basis of the estimated GFR rather than serum
creatinine.
Dosing UFH 3 times daily for VTE prophylaxis or using LMWH.
Treating severe CDAD with oral vancomycin.
Prescribing annual zolendronic acid for hip fracture patients unable or
unwilling to use oral bisphosphonates.
Using pre-extubation steroids to prevent tracheal edema and
reintubation.
Acute phase anticoagulation for suspected acute cardioembolic stroke.
Consider $\quad$ Using intravenous bicarbonate plus NAC for CIN prophylaxis.
Adding tolvaptan to standard therapy for acute decompensated heart
failure. Select patients who closely mirror those in the EVEREST trial.
Stopping combined warfarin/aspirin for secondary cardiovascular
prevention except among patients with mechanical heart valves.

Abbreviations: CDAD, Clostridium difficile-associated diarrhea; CIN, contrast-induced nephropathy; EVEREST, Efficacy of Vasopressin Antagonism in Heart Failure Outcome Study with Tolvaptan; GFR, glomerular filtration rate; LMWH, low-molecular-weight heparin; NAC, N-acetylcysteine; UFH, unfractionated heparin; VTE, venous thromboembolism.

in this prospective multihospital registry. Excess dosing was associated with substantially higher rates of major in-hospital bleeding and death, with a number needed to harm of 78 for major bleeding and a number needed to harm of 167 for in-hospital death. In comparison, the number needed to treat with another low-molecular-weight heparin (dalteparin) was 34 to prevent 1 death or myocardial infarction in the first 6 days, with a nonsignificant trend toward decreased mortality. ${ }^{1}$

Commentary: Providers likely underestimate the degree of renal impairment when looking solely at serum creatinine instead of estimates of $\mathrm{CrCl}$. Excess dosing was more common among elderly, thin, and female patients. Clinicians must calculate the enoxaparin dose on the basis of careful estimates of $\mathrm{CrCl}$ to limit this risk. The Modification of Diet in Renal Disease (MDRD) equation is commonly used for this purpose.

Clinical Bottom Line: Enoxaparin excess dosing is common and harmful. Clinicians can mitigate this risk by more carefully estimating renal function when selecting the proper enoxaparin dose of 1 $\mathrm{mg} / \mathrm{kg}$ twice daily for $\mathrm{CrCl} \geq 30 \mathrm{~mL} / \mathrm{minute}$ and 1 $\mathrm{mg} / \mathrm{kg}$ once daily for $\mathrm{CrCl}<30 \mathrm{~mL} / \mathrm{minute}$.

\section{Venous Thromboembolism Prevention}

Wein L, Wein S, Haas SJ, et al. Pharmacological venous thromboembolism prophylaxis in hospitalized medical patients. Arch Intern Med. 2007;167:1476-1486.

Question: What is the relative safety and efficacy of various pharmacological agents for preventing venous thromboembolism among hospitalized medical patients?

Sponsor: National Health and Medical Council of Australia.

Study Design: Meta-analysis of 36 prospective randomized controlled trials involving about 48,000 patients.

Study Selection: Prospective randomized controlled trials enrolling at least 30 patients comparing 1 of 4 regimens: (1) unfractionated heparin (UFH) versus control, (2) low-molecular-weight heparin (LMWH) versus control, (3) LMWH versus UFH, or (4) Factor Xa inhibitor versus placebo. Trials of surgical, trauma, and critical care patients were excluded. Only 1 Factor Xa trial (fondaparinux) was located, ${ }^{2}$ and thus it was not eligible for meta-analysis.

Outcomes: Pooled relative risks with $95 \%$ confidence intervals for deep venous thrombosis (DVT), pulmonary embolism (PE), mortality, and total bleeding. The authors also compared 2 UFH regimens: 5000 units twice daily versus 5000 units thrice daily.

Results: UFH (all doses, compared with control): The relative risk was 0.33 (95\% CI $0.26-0.42)$ for DVT and 0.64 (95\% CI $0.50-0.82)$ for PE $(P=0.001$ for both). Mortality was not different. The relative risk for major bleeding was 3.11 (95\% CI 2.443.96, $P=0.001)$.

LMWH (compared with control): The relative risk was $0.56(95 \%$ CI $0.45-0.70)$ for DVT and 0.37 (95\% CI $0.21-0.64)$ for PE ( $P=0.001$ for both). Mortality was not different. The relative risk for major bleeding was 1.92 (95\% CI 1.32-2.78, $P=$ $0.001)$.

LMWH (compared with UFH, all doses): The relative risk for DVT was 0.68 (95\% CI $0.52-0.88$, $P=0.004)$, but the risk was not different for PE, mortality, or major bleeding.

UFH (5000 units twice daily, compared with control): The relative risk for DVT was $0.52(95 \%$ CI $0.28-0.96, P=0.04)$. When the random-effects model was used, this difference became statistically nonsignificant (relative risk $=0.41,95 \% \mathrm{CI}$ 0.10-1.73, $P=0.23)$.

UFH (5000 units 3 times daily, compared with control): The relative risk for DVT was $0.27(95 \%$ CI $0.20-0.36, P=0.001$ ). This difference remained when the random-effects model was applied (relative risk $=0.28,95 \%$ confidence interval $=0.21-$ $0.38, P=0.001$ ). 
Conclusions: Both UFH and LMWH reduce DVT and PE in hospitalized medical patients. Neither affects mortality. Both increase the risk of major bleeding. LMWH reduces the risk of DVT but not the risk of PE in comparison with UFH (all doses). When adjusted for random effects, UFH at a dose of 5000 units twice daily does not appear to be different than the control.

Commentary: This well-conducted meta-analysis demonstrates the efficacy of heparin, whether unfractionated or low-molecular-weight, in the prevention of venous thromboembolism. Of note, the UFH dose of 5000 units twice daily did not appear to be different than placebo. The UFH dose of 5000 units 3 times daily, by contrast, was effective in both the fixed-effects and randomeffects models. Mortality was unaffected by any of the regimens studied. All regimens were associated with increased risks of major bleeding.

Clinical Bottom Line: Pharmacological prophylaxis with UFH 3 times daily or LMWH reduces the risk for venous thromboembolism. Twice daily UFH is not clearly different from placebo. Overall mortality was unaffected by any of the regimens for prophylaxis.

\section{Contrast Nephropathy Prevention}

Briguori C, Airoldi F, D'Andrea D, et al. Renal insufficiency following contrast media administration trial (REMEDIAL): a randomized comparison of 3 preventive strategies.

Circulation. 2007;115:1211-1217.

Question: What is the efficacy of saline versus bicarbonate for the prevention of contrast mediainduced nephropathy?

Sponsor: Institutional funding (C. Briguori, personal communication, January 2008).

Study Design: Randomized trial.

Patients: Three hundred twenty-six consecutive patients with serum creatinine $\geq 2.0 \mathrm{mg} / \mathrm{dL}$ and/ or an estimated glomerular filtration rate $<40$ $\mathrm{mL} /$ minute $/ 1.73 \mathrm{~m}^{2}$ undergoing elective coronary and/or peripheral angiography.

Setting: Two interventional cardiology laboratories in Italy.

Intervention: Patients were randomized to 1 of 3 preventive regimens: (1) intravenous saline $(0.9 \%)$ given at a rate of $1 \mathrm{~mL} / \mathrm{kg}$ of body weight/hour 12 hours prior to the procedure and continuing for 12 hours afterward (reduced to $0.5 \mathrm{~mL} / \mathrm{kg} /$ hour for patients with a left ventricular ejection fraction < $40 \%$ ) plus $\mathrm{N}$-acetylcysteine (NAC; $1200 \mathrm{mg}$ orally twice daily) on the day before the procedure and the day of the procedure; (2) intravenous sodium bicarbonate $(154 \mathrm{mEq} / \mathrm{L}$ in dextrose and water) given as an initial bolus of $3 \mathrm{~mL} / \mathrm{kg}$ over 1 hour prior to the procedure and continuing at a rate of 1 $\mathrm{mL} / \mathrm{kg} /$ hour for 6 hours more plus NAC as above; or (3) intravenous saline as above plus intravenous ascorbic acid ( $3 \mathrm{~g}) 2$ hours prior to the procedure followed by $2 \mathrm{~g}$ on the night and morning after the procedure plus NAC as above.

Outcomes: Rate of contrast-induced nephropathy (CIN), which was defined as an increase in serum creatinine $\geq 25 \%$ from the baseline value at 48 hours after the administration of contrast or the need for hemodialysis.

Follow-Up: Forty-eight hours.

Results: The baseline serum creatinine was about $2.0 \mathrm{mg} / \mathrm{dL}$ and did not differ among the 3 groups. The rate of CIN was $9.9 \%(11 / 111)$ in the saline plus NAC group, $1.9 \%(2 / 108)$ in the bicarbonate plus NAC group, and $10.3 \%(11 / 107)$ in the saline plus ascorbic acid plus NAC group. The bicarbonate plus NAC regimen was superior to saline plus NAC $(P=0.019)$. The absolute risk reduction for bicarbonate plus NAC versus saline plus NAC was $8 \%$ (a number needed to treat of 13 to prevent 1 case of CIN). The saline plus NAC and saline plus ascorbic acid plus NAC groups did not differ in outcome.

Conclusions: Sodium bicarbonate plus NAC is superior to saline plus NAC for the prevention of CIN among patients with baseline chronic kidney disease.

Commentary: This trial confirms the results of the initial study by Merten et al. ${ }^{3}$ showing the superiority of bicarbonate versus saline in the prevention of CIN. That trial, published in 2004, did not use NAC. Also in 2007, 3 other single-center randomized trials of saline versus bicarbonate in the prevention of CIN were published. ${ }^{4-6}$ All concluded that bicarbonate is superior to saline. Whether NAC is effective for CIN prevention remains unclear. ${ }^{7}$ Given its low side-effect profile, it is not unreasonable to continue using NAC until further data are available. At-risk patients receiving intravenous contrast for other indications (eg, computed tomography) would likely show similar benefit. Although there are now 5 prospective blinded controlled trials showing the superiority of bicarbonate, a recently published large retrospective cohort found that the use of sodium bicarbonate was associated with increased incidence of $\mathrm{CIN}^{8}$ The concordant 
results of all 5 prospective randomized trials of sodium bicarbonate, along with the risk for unmeasured confounding variables with retrospective cohort analysis, suggest that bicarbonate is superior to saline in the prevention of CIN.

Clinical Bottom Line: Clinicians should consider selecting intravenous bicarbonate rather than saline for the prevention of CIN.

\section{Acute Decompensated Heart Failure Treatment Gheorghiade M, Konstam MA, Burnett JC, et al.}

Short-term clinical effects of tolvaptan, an oral vasopressin antagonist, in patients hospitalized for heart failure: the EVEREST clinical status trials. JAMA. 2007;297:1332-1343.

Question: What is the efficacy and safety of shortterm tolvaptan added to standard therapy in the treatment of acute decompensated heart failure?

Sponsor: Otsuka America, Inc.

Study Design: Two concurrent randomized, double-blind, placebo-controlled trials. Two trials (each with different sites) were conducted to "fulfill regulatory requirements for establishing efficacy from at least 2 independent, adequately powered, and well-controlled trials."

Patients: Two thousand forty-eight adults (trial A) and 2085 adults (trial B) hospitalized with heart failure. Eligibility criteria included a history of chronic heart failure requiring treatment for at least 30 days prior to admission, an ejection fraction $\leq 40 \%$ at any point in the prior year, dyspnea at rest or with minimal exertion, and 2 or more signs of congestion (dyspnea, jugular vein distension, or peripheral edema). Selected exclusionary criteria included active myocardial ischemia, recent cardiac surgery, systolic blood pressure $<90 \mathrm{~mm} \mathrm{Hg}$, serum creatinine $>3.5 \mathrm{mg} / \mathrm{dL}$, serum potassium $>5.5 \mathrm{mg} / \mathrm{dL}$, or hemoglobin $<9 \mathrm{~g} / \mathrm{dL}$.

Setting: Three hundred fifty-nine sites across North America, South America, and Europe. Trial A patients were assigned from 179 of these sites. Trial B patients were assigned from 180 of these sites.

Intervention: Tolvaptan, a vasopressin antagonist (30 mg orally daily), versus matching placebo, in addition to standard therapy. Treatment was started within 48 hours of admission and was continued through discharge for a minimum of 60 days.

Outcomes: Composite of global clinical status and body weight at day 7 or at discharge if earlier. Additional secondary endpoints were dyspnea (day 1) and peripheral edema (day 7).

Follow-Up: Seven days.
Results: Tolvaptan improved the composite primary endpoint compared with placebo, and this was primarily related to greater overall net diuresis: $3.35 \mathrm{~kg}$ of diuresis at day 7 or discharge with tolvaptan versus $2.73 \mathrm{~kg}$ with placebo (trial A) and $3.77 \mathrm{~kg}$ of diuresis at day 7 or discharge with tolvaptan versus $2.79 \mathrm{~kg}$ with placebo (trial $\mathrm{B} ; P<$ 0.001 for both trials). Net diuresis at day 1 was also greater with tolvaptan. More patients reported improved dyspnea at day 1 with tolvaptan: $76.74 \%$ versus $70.61 \%$ (trial A) and $72.06 \%$ versus $65.32 \%$ (trial B; $P<0.001$ for both comparisons). Edema scores at day 7 favored tolvaptan in trial B $(P=$ $0.02)$ but in not trial A $(P=0.07)$. Hypernatremia was more common with tolvaptan in trial A $(1.4 \%$ versus $0 \%, P<0.001)$ but not in trial B $(0.5 \%$ versus $0 \%, P=0.06)$. Tolvaptan-treated patients had lower average furosemide doses than placebotreated patients. Patient-assessed global clinical status at day 7 , as measured by a visual analog scale, was no different.

Conclusions: Tolvaptan, added to standard care for acute heart failure, safely improved many but not all short-term heart failure signs and symptoms.

Commentary: The accompanying Efficacy of Vasopressin Antagonism in Heart Failure Outcome Study with Tolvaptan (EVEREST) outcomes trial demonstrated that longer term use of tolvaptan for 60 days was not associated with changes in cardiovascular morbidity and mortality. ${ }^{9}$ Concerns have been raised about the safety of nesiritide $\mathrm{e}^{10}$ and inotropes ${ }^{11}$ in the treatment of acute decompensated heart failure. With the completion of this 2-part trial, we have a safe addition to the current armamentarium of treatments for acute decompensated heart failure. Clinicians should exercise caution in adding tolvaptan only to patients whose characteristics mirror those in this trial.

Clinical Bottom Line: Tolvaptan represents an effective and safe addition to therapies for acute decompensated heart failure.

\section{Cardiovascular Risk Reduction}

Dentali F, Douketis JD, Lim W, Crowther M. Combined aspirin-oral anticoagulant therapy compared with oral anticoagulant therapy alone among patients at risk for cardiovascular disease: a meta-analysis of randomized trials. Arch Intern Med. 2007;167:117-124.

Question: For patients receiving oral anticoagulant therapy (OAC), does the addition of aspirin reduce major adverse cardiovascular endpoints? 
Sponsor: Heart and Stroke Foundation of Canada. Study Design: Meta-analysis of 10 randomized controlled trials.

Study Selection: From MEDLINE (to June 2005), EMBASE (to June 2005), and Cochrane (to 2005, issue 2) reviews, including manual reference list reviews, 10 studies were identified that satisfied 4 criteria: (1) a randomized controlled trial in patients requiring OAC therapy, (2) a comparison of combined aspirin-OAC therapy with OAC alone (the same target international normalized ratio in both arms), (3) follow-up of at least 3 months, and (4) at least 1 prespecified outcome that was objectively documented. The 10 trials meeting these criteria studied 4180 patients. The target international normalized ratio varied across the trials on the basis of the population studied. The aspirin dose was at least $75 \mathrm{mg} /$ day in all studies.

Outcomes: Arterial thromboembolism, all-cause mortality, and major bleeding. Secondary outcomes included fatal arterial thromboembolism and fatal major bleeding.

Results: Arterial thromboembolism was lower with combined aspirin-OAC therapy $(6.3 \%)$ versus OAC therapy alone $(8.8 \%$; absolute risk reduction $=2.5 \%$, number needed to treat $=40, P<0.001$ ). In subgroup analysis, this difference was found only among patients with mechanical heart valves (odds ratio $=0.27,95 \%$ CI $0.15-0.49$ ). There was no benefit among patients with atrial fibrillation (odds ratio $=0.99,95 \%$ CI $0.47-2.07$ ) or coronary artery disease (odds ratio $=0.69,95 \%$ CI 0.35-1.3). Mortality was no different. Major bleeding was more common with combined therapy $(3.8 \%)$ versus OAC therapy alone $(2.8 \%$; absolute risk reduction $=1.0 \%$, number needed to harm $=100, P=0.05)$. Secondary outcomes were not different.

Conclusions: Combined aspirin-OAC therapy does not protect against future arterial thromboembolism in comparison with OAC therapy alone, except among patients with mechanical heart valves. Combined therapy, however, is associated with higher rates of major bleeding.

Commentary: These findings question the current practice of combining OAC with aspirin in patients with separate indications for each. Looking in more detail at the analyzed trials, the researchers found that there were relatively few patients with proven coronary artery disease. There may have been insufficient power to show a benefit for combined therapy among these patients. Patients with mechanical heart valves, however, clearly showed benefit. A recently published retrospective study of more than 4000 patients also concluded that the hemorrhagic risk of combined aspirin-OAC therapy versus OAC therapy alone appeared to outweigh the benefit. $^{12}$

Clinical Bottom Line: Except among patients with mechanical heart valves, combined aspirin-OAC increases bleeding risk without proven benefit. Until further data are available, clinicians should individualize antithrombotic therapy on the basis of a careful assessment of risk and benefit.

\section{Cardioembolic Stroke Treatment}

Paciaroni M, Agnelli G, Micheli S, Caso V. Efficacy and safety of anticoagulant treatment in acute cardioembolic stroke: a meta-analysis of randomized controlled trials.

Stroke. 2007;38:423-430.

Question: What are the safety and efficacy of anticoagulation in the treatment of acute cardioembolic stroke?

Sponsor: None.

Study Design: Meta-analysis of 7 randomized controlled trials.

Study Selection: Trials randomizing patients within 48 hours from stroke onset with objectively diagnosed stroke of presumed cardioembolic origin that compared full-dose anticoagulants (unfractionated heparin, low-molecular-weight heparin, and heparinoid) to other treatments (aspirin or placebo) for initial therapy and used objective methods to assess study outcomes.

Outcomes: A composite of death or disability at final follow-up (at least 3 months), all new strokes (ischemic and hemorrhagic) at 14 days, and pulmonary embolism. The safety outcome was symptomatic intracranial bleeding.

Results: The odds ratio (95\% CI) for death or disability with anticoagulation versus aspirin or placebo was 1.01 (95\% CI 0.82-1.24); the odds ratio for all new strokes with anticoagulation versus aspirin or placebo was 1.18 (95\% CI $0.74-1.88$ ). The odds ratio for pulmonary embolism with anticoagulation versus aspirin was 0.94 (95\% CI $0.44-$ $2.00)$. None of these were statistically significant. However, the odds ratio for symptomatic intracranial hemorrhage with anticoagulation versus aspirin or placebo was 2.89 (95\% CI 1.19-7.01, $P=$ 0.02). The absolute increase in symptomatic intracranial bleeding with anticoagulation was $1.8 \%$ 
(number needed to harm $=55$ ). Of the 7 trials analyzed, 1 trial did show a reduction in overall death or disability with anticoagulation, in which therapy was started within 3 hours of symptom onset (odds ratio $=0.49,95 \%$ CI $0.26-0.93$ ). This trial was small, and subgroup analysis in the other, larger trials failed to confirm this finding.

Conclusion: Anticoagulation for acute stroke of suspected cardioembolic origin does not improve outcomes but is associated with higher rates of symptomatic intracranial hemorrhage.

Commentary: Long-term anticoagulation with sodium warfarin clearly lowers cardioembolic stroke risk for patients with chronic atrial fibrillation. This meta-analysis demonstrates that acute anticoagulation does not reduce the composite endpoint of death or disability, recurrent stroke, or pulmonary embolism. The risk of symptomatic intracranial hemorrhage is substantially increased and argues against the use of anticoagulants during the acute phase of suspected cardioembolic stroke.

Clinical Bottom Line: Anticoagulation is harmful and does not reduce death or disability in the acute phase of suspected cardioembolic stroke.

\section{Clostridium Difficile-Associated Diarrhea Zar FA, Bakkanagari SR, Moorthi KM, Davis MB. \\ A comparison of vancomycin and metronidazole for the treatment of Clostridium difficile-associated diarrhea, stratified by disease severity. Clin Infect Dis. \\ 2007;45:302-307.}

Question: What is the best first-line treatment for Clostridium difficile-associated diarrhea (CDAD)?

Sponsor: None.

Study Design: Randomized, double-blind, placebo-controlled trial.

Patients: One hundred fifty patients with 3 or more nonformed stools in 24 hours with a positive stool $C$. difficile toxin A test or the presence of pseudomembranous colitis on endoscopy.

Setting: A 200-bed community teaching hospital affiliated with an academic medical center.

Intervention: Metronidazole (250 mg 4 times daily) plus vancomycin liquid placebo versus metronidazole placebo plus vancomycin liquid (125 mg 4 times daily), both for 10 days.

Outcomes: The primary outcomes were cure (resolution of diarrhea by day 6 of treatment and a negative stool toxin at both 6 and 10 days post- treatment), treatment failure (persistent diarrhea and/or an inability to clear the toxin at 6 days, the need for colectomy, or death after 5 days of treatment), and relapse (recurrence of toxin-positive CDAD by day 21 after the initial cure). Disease was categorized as mild $(<2$ points $)$ or severe $(\geq 2$ points), with 1 point each for age $>60$ years, temperature $>38.3^{\circ} \mathrm{C}$, albumin $<2.5 \mathrm{mg} / \mathrm{dL}$, and a peripheral white blood count $>15,000$ cells $/ \mathrm{mm}^{3}$ within 48 hours of enrollment. Two points were allotted for endoscopic findings of pseudomembranous colitis.

Follow-Up: Patients were monitored for 21 days for resolution of diarrhea ( $\leq 2$ formed stools in 24 hours). Stool toxin was measured at days 6 and 10 of treatment and at day 21 if diarrhea was still present.

Results: One hundred fifty patients (81 patients with mild disease and 69 patients with severe disease) finished the trial, with no significant differences in patients categorized into the 2 treatment arms. Overall, $84 \%(66 / 79)$ of patients receiving metronidazole were cured versus $97 \%(69 / 71)$ of patients receiving vancomycin $(P=0.006)$. In patients with mild disease, $90 \%(37 / 41)$ and $98 \%$ $(39 / 40)$ were cured in the metronidazole-treated and vancomycin-treated groups, respectively $(P=$ 0.36). In patients with severe disease, $76 \%(29 / 38)$ and $97 \%(30 / 31)$ were cured in the metronidazoletreated and vancomycin-treated groups, respectively $(P=0.02)$. After the initial cure, relapse occurred in $7 \%(5 / 76), 15 \%(9 / 59), 14 \%(9 / 66)$, and $7 \%(5 / 69)$ of patients with mild disease, severe disease $(P=0.15$ for mild versus severe), metronidazole treatment, and vancomycin treatment ( $P=0.27$ between treatments), respectively. In patients with severe CDAD, low albumin, intensive care, and presence of pseudomembranous colitis were associated with metronidazole treatment failure.

Conclusion: Metronidazole is equally effective as vancomycin in treating mild CDAD; however, vancomycin appears superior to metronidazole in treating patients with severe CDAD.

Commentary: Two prior studies evaluating metronidazole and vancomycin for CDAD revealed no significant difference between the 2 therapies. ${ }^{13,14}$ However, these studies had serious methodological flaws, including a lack of blinding and too little power to show a difference. This randomized, double-blind, placebo-controlled trial provides convincing evidence that oral vancomycin is 
superior to metronidazole in patients with severe CDAD. This is an especially important finding as the recently described hypervirulent epidemic strain of $C$. difficile becomes more prevalent.

A single-center retrospective study of 102 veterans with metronidazole-treated CDAD showed analogous findings with a slightly different scoring system. ${ }^{15}$ In $94 \%$ of metronidazole responders, the score was 2 or less. In $67 \%$ of true failures, the score was greater than 2. Taken together, these studies suggest that higher scores predict metronidazole failure.

Clinical Bottom Line: Vancomycin appears to be more effective than metronidazole in treating more severe forms of CDAD.

\section{Consultative Medicine: Orthopedics Lyles KW, Colón-Emeric CS, Magaziner JS, et al. Zoledronic acid and clinical fractures and mortality after hip fracture. N Engl J Med. 2007;357:1799-1809.}

Question: Does an annual dose of zoledronic acid reduce the rate of subsequent fractures and mortality in patients with a recent hip fracture?

Sponsor: Novartis.

Study Design: Placebo-controlled, double-blinded, randomized controlled trial.

Patients: A total of 2127 men and women 50 years old or older with a surgically repaired low-impact hip fracture (eg, fall from a standing height) within 90 days of study entry who were unwilling or unable to take an oral bisphosphonate.

Setting: International and multicenter.

Intervention: A single 5-mg intravenous dose of zoledronic acid within 90 days of a hip fracture repair versus an intravenous placebo, given annually. All patients with documented vitamin D deficiency or no documentation of a serum 25hydroxyvitamin $\mathrm{D}$ level received a loading dose of vitamin $\mathrm{D}_{3}$ or $\mathrm{D}_{2} 14$ days prior to the first infusion. All patients received oral calcium and vitamin D daily after the first infusion.

Outcomes: The primary outcome was a new clinical fracture excluding facial, digital, or abnormal bone (eg, bone with metastases) fractures. Secondary outcomes included changes in the bone mineral density in the nonfractured hip, the number of new vertebral, nonvertebral, and hip fractures, and predetermined safety outcomes.

Follow-Up: Quarterly phone calls and annual clinic visits for up to 5 years.
Results: The trial was stopped early after prespecified efficacy objectives were met. At an average follow-up of 1.9 years, $8.6 \%$ of subjects receiving zoledronic acid and $13.9 \%$ of those receiving placebo suffered subsequent fractures $(P=0.001)$. Statistically significant improvements in bone mineral density were seen at both the total hip and femoral neck sites in the zoledronic acid group versus the placebo group. Approximately $80 \%$ of patients experienced an adverse event in each group, with statistically significantly more pyrexia, myalgias, and bone pain in the zoledronic acid cohort and higher mortality in the placebo group, that is, $9.6 \%$ versus $13.3 \%$ (hazard ratio $=0.72,95 \%$ CI 0.56-0.72, $P=0.01$ ).

Conclusion: Annual treatment with $5 \mathrm{mg}$ of intravenous zoledronic acid reduces clinical fractures and mortality when it is dosed within 90 days of a hip fracture repair.

Commentary: Patients who suffer a hip fracture are at high risk for successive fractures, with a considerable morbid and financial burden on the patient and the healthcare system. Additionally, as many as 1 in 4 of these patients will die in the subsequent year. Poor adherence to oral bisphosphonates and prescriber nonadherence to fracture guidelines are common sources of noncompliance and have been associated with increased fracture burden. The findings that an annual infusion can achieve reductions in the fracture rate and mortality are notable and offer options for patients who otherwise could not comply with therapy because of side effects or an inability to take a more frequently dosed medication.

Clinical Bottom Line: An annual dose of zoledronic acid reduces the rate of subsequent fractures and death in patients with a recent hip fracture.

\section{Critical Care Medicine}

Francois B, Bellissant E, Gissot V, et al. 12-h pretreatment with methylprednisolone versus placebo for prevention of postextubation laryngeal edema: a randomized double blind trial. Lancet. 2007;369:1083-1089.

Question: Do pre-extubation steroids prior to planned extubation prevent postextubation laryngeal edema?

Sponsor: Institutional funding (P. Vignon, personal communication, March 2008).

Study Design: Placebo-controlled, double-blinded, randomized controlled trial. 
Patients: Seven hundred sixty-one adult patients with at least 36 hours of mechanical ventilation and planned extubation.

Setting: Fifteen intensive care units in France.

Intervention: Intravenous methylprednisolone (20 mg) starting 12 hours before extubation and continuing every 4 hours until extubation, including the time of extubation (total dose $=80 \mathrm{mg}$ ), or a placebo identical in appearance and delivery.

Outcomes: The primary outcome was the development of minor (inspiratory stridor associated with respiratory distress requiring intervention) or major (reintubation secondary to laryngoscopically visualized upper airway obstruction) laryngeal edema within 24 hours of extubation.

Follow-Up: Clinical assessments were performed 10 minutes and $1,1.5,3,6,12$, and 24 hours after extubation.

Results: Six hundred ninety-eight patients completed the trial. The median duration of intubation prior to extubation was 6 days. Any laryngeal edema occurred in $22 \%(76 / 343)$ and $3 \%(11 / 355)$ of patients in the placebo and treatment groups, respectively $(P<0.0001)$. When edema was present, the severity and timing of the onset of edema did not differ between the 2 groups. Reintubation was reduced from $8 \%(26 / 343)$ in the placebo group to $4 \%(13 / 355)$ in the treatment groups $(P$ $=0.02)$. When necessary, reintubation was deemed secondary to major edema in $54 \%(14 / 26)$ of the placebo group and $8 \%(1 / 13)$ of the treatment group, respectively. An intention-to-treat analysis did not alter the study findings. One patient in each group suffered a serious adverse event: respiratory failure and death 23 hours after extubation in the placebo group and septic shock and death 26 hours after extubation in the treatment group. Rates of hyperglycemia and infections were not reported.

Conclusion: The use of 20-mg intravenous doses of methylprednisolone spaced 4 hours apart and starting 12 hours prior to planned extubation is associated with significant reductions in the rates of tracheal edema and reintubation.

Commentary: Postextubation laryngeal edema is common (2\%-22\% incidence) and results in reintubation for $0.7-4.7 \%$ of extubations. This work shows that a simple pretreatment with intravenous steroids 12 hours before planned extubation can reduce the rate of postextubation edema 7fold, including a 2 -fold reduction in the reintubation rate. Prior trials using shorter periods of treat- ment $(<6$ hours) have not shown benefit, so achieving this study's results likely requires the full 12-hour protocol.

Clinical Bottom Line: Intravenous methylprednisolone dosed 12 hours before and every 4 hours until planned extubation reduces the rate of reintubation due to tracheal edema.

Address for correspondence and reprint requests: Mel L. Anderson, Department of Medicine, Denver VA Medical Center, 1055 Clermont Street, Denver, CO 80220; Telephone: 303-399-8020, ext. 2396; Fax: 303-393-5199; E-mail: melver.anderson@va.gov

Received 7 March 2008; revision received 8 May 2008; accepted 26 May 2008.

\section{REFERENCES}

1. Fragmin During Instability in Coronary Artery Disease (FRISC) Study Group. Low molecular weight heparin during instability in coronary artery disease. Lancet. 1996;347:561-568.

2. Cohen AT, Davidson BL, Gallus AS, et al. Efficacy and safety of fondaparinux for the prevention of venous thromboembolism in older acute medical patients: randomised placebo controlled trial. BMJ. 2006;332:325-329.

3. Merten GJ, Burgess WP, Gray LV, et al. Prevention of contrast-induced nephropathy with bicarbonate: a randomized controlled trial. JAMA. 2004;291:2328-2334.

4. Recio-Mayoral A, Chaparro M, Prado B, et al. The renoprotective effect of hydration with sodium bicarbonate plus $\mathrm{N}$-acetylcysteine in patients undergoing emergency percutaneous interventions: the RENO study. J Am Coll Cardiol. 2007;49:1283-1288.

5. Masuda M, Yamada T, Mine T, et al. Comparison of usefulness of sodium bicarbonate versus sodium chloride to prevent contrast-induced nephropathy in patients undergoing an emergent coronary procedure. Am J Cardiol. 2007;100: 781-786.

6. Ozcan EE, Guneri S, Akdeniz B, et al. Sodium bicarbonate, $\mathrm{N}$-acetylcysteine and saline for the prevention of radiocontrast-induced nephropathy. A comparison of 3 regimens for protecting contrast-induced nephropathy in patients undergoing coronary procedures. A single-center prospective controlled trial. Am Heart J. 2007;154:539-544.

7. Bagshaw SM, McAlister FA, Manns BJ, Ghali WA. Acetylcysteine in the prevention of contrast-induced nephropathy: a case study of the pitfalls in the evolution of evidence. Arch Intern Med. 2006;166:161-166.

8. From AM, Bartholmai BJ, Williams AW, et al. Sodium bicarbonate is associated with an increased incidence of contrast nephropathy: a retrospective cohort study of 7977 patients at Mayo Clinic. Clin J Am Soc Nephrol. 2008; 3:10-18.

9. Konstam MA, Gheorghiade M, Burnett JC, et al. Short-term clinical effects of tolvaptan, an oral vasopressin antagonist, in patients hospitalized for heart failure: the EVEREST clinical status trials. JAMA. 2007;297:1332-1343.

10. Sackner-Bernstein JD, Kowalski M, Fox M, Aaronson A. Short-term risk of death after treatment with nesiritide for acute decompensated heart failure: a pooled analysis 
of randomized controlled trials. JAMA. 2005;293:19001905.

11. Cuffe MS, Califf RM, Adams KF Jr, et al. Short-term intravenous milrinone for acute exacerbations of chronic heart failure: a randomized controlled trial. JAMA. 2002;287: $1541-1547$

12. Johnson SG, Rogers K, Delate T, Witt DM. Outcomes associated with combined antiplatelet and anticoagulant therapy. Chest. 2008;133:948-954.

13. Teasley DG, Gerding DN, Olson MM, et al. Prospective randomized trial of metronidazole versus vancomycin for
Clostridium difficile-associated diarrhea and colitis. Lancet. 1983;2:1043-1046.

14. Wenisch C, Parschalk B, Hasenhundl M, Hirschl AM, Graninger W. Comparison of vancomycin, teicoplanin, metronidazole, and fusidic acid for the treatment of Clostridium difficile-associated diarrhea. Clin Infect Dis. 1996;22: 831-838.

15. Belmares J, Gerding DN, Parada JP, et al. Outcome of metronidazole therapy for Clostridium difficile disease and correlation with a scoring system. J Infect. 2007;55: 495-501. 